\title{
A Cross Layer based Vertical Handoff Decision Making Framework
}

\author{
A. Bhuvaneswari \\ Department of Computer Science, \\ Cauvery College for Women, Trichy, Tamil Nadu, \\ India.
}

\author{
E. George Dharma Prakash Raj \\ Phd,Department of Computer Science, \\ BharathidasanUniversity, Trichy, Tamil Nadu, India
}

\begin{abstract}
Emerging wireless communication technology focuses the development to provide the communication support for mobile devices based on the user demand and network connectivity for high-speed data transfer like multimedia, video and Voice over IP(VoIP). In wireless network the main issue to be addressed is channel allocation and network connectivity. Because of the limited bandwidth resources, it is necessary to provide an efficient solution for bandwidth allocation to increase number of users. Maintaining the network connectivity improves the user satisfaction. Because of the mobility of user and continuous changes in network characteristics like bandwidth, error rate, delay and jitter assigning the 'best' network channel is a challenging issue. Handover process has been done to maintain the connectivity. During handover choosing the best network with the QoS guarantee based on the user requirements and service type is the current research topic. Several works is going on in this area. Still there is no standard and efficient method implemented in real world. This paper proposes a frame work to make decision to choose the best network while handoff is triggered.
\end{abstract}

Keywords - Vertical Handoff Decision Making, Cross layer, QoS, Wi-Fi, Wi-Max, GPRS, CDMA

\section{INTRODUCTION}

The current advancement and demand in wireless communication technology, and the requirement of QoS with ubiquitous feature, motivates the researchers to identify the solution to meet the demand. The main focus in mobile communication is to provide the seamless mobility and service continuity. Mobility management plays an important role to have a successful wireless communication which can be divided into Location Management and Handover Management. Location Management tracks the location of the mobile terminals to have consecutive communication. Handoff Management is the process in which mobile terminal(MT) keeps its connection active when is moves from one access point to another. Normally, handoff can be categorized as intra-system or Horizontal Handoff, which is handoff between same type(homogeneous) of networks like WLAN to WLAN, Wi-Fi to Wi-Fi etc., and inter-system or Vertical Handoff, which is handoff between different networks(heterogeneous) like WLAN to Wi-Fi, Wi-Fi to WiMax etc., The current development of wireless communication technology offers multi-mode interfaces to access a wide range of services provided by multiple wireless networks such as Bluetooth, Wi-Fi, Wi-Max, GPRS and CDMA. Each one is developed to meet specific service requirements with its own merits and demerits. Each network has different characteristics and deciding the best one which suits for the current service requirements, user requirements with best Quality of Service (QoS) is an important issue for Vertical Handoff.
Table I. Different Network Characteristics [1]

\begin{tabular}{|c|c|c|c|}
\hline Network Type & Coverage & Bandwidth & Cost \\
\hline Wi-Fi & $\begin{array}{l}\text { Shorter } \\
\text { Range }\end{array}$ & High & Low \\
\hline UMTS & $\begin{array}{l}\text { Larger } \\
\text { distance }\end{array}$ & Limited & Expensive \\
\hline Wi-Max & $\begin{array}{l}\text { Medium } \\
\text { Range }\end{array}$ & Medium & High \\
\hline Satellite & $\begin{array}{l}\text { World- } \\
\text { Wide }\end{array}$ & High & Very High \\
\hline
\end{tabular}

This paper is organized as follows: Section II discuss about the basic concepts of handoffs. Section III explains the existing approaches for vertical handoff decision making. Section IV list outs the parameter to be considered for decision making and performance. Section V depicts the proposed framework for vertical handoff decision making and its explanation. Section VI concludes the paper.

\section{HANDOFF BASICS}

The handover process which minimizes or eliminates the delay for establishing a new connection with a BS is called a fast handover, and when the data loss is minimized it is named a smooth handover. Combination of these qualities is called a seamless handover. Seamless handover features are tolerable delay, maximum throughput and low packet loss with eliminating the ping pong effect which is caused by frequent handoff.

Handoff can be classified as Horizontal Handoff which is handoff between same network and Vertical Handoff which handoff between different networks. If the connection with the old BS is broken before established with the new BS it is called as Hard Handover. In a Soft Handover the connection is made with the new BS before the old one is lost. The direction of handover process is categorized as Upward vertical handover in which MN switches from a smaller coverage, high bandwidth network to a larger coverage and low bandwidth network. The Downward vertical handoff is just a vice versa.

When the mobile node is controlled based by the network based on the information about its network condition it is called network controlled. Otherwise, if the mobile node controlled by mobile device itself based the information about all the networks it is called mobile controlled. Mobile Node receives information of all networks because of its multimode interface capability. Imperative handover takes place based on the MN connection. Alternative handover takes place when there is a need for user preferences. Imperative can be reactive or proactive. Reactive decisions are mainly up on current network information only. Proactive decision attempts to predict future conditions based on knowledge based and modeling based. Pre-recorded information is used 
by the Knowledge based handover and modeling based approach predicts future conditions with mathematical models. Following figure depicts the categories of Vertical Handoff [2].

\section{VERTICAL HANDOVER DECISION MAKING PROCESS AND RELATED \\ WORK}

Vertical Handover Decision Making process is divided into three phases [3].

Information Gathering Module: This module collects the information about user preferences, network conditions to decide when to initiate handoff triggering event.
Handoff Decision Module: This module is responsible to select the best network based on the requirement of user and service by applying decision making algorithm immediately when the handoff trigger event occurs.

Handoff Execution: Mobile Node is assigned with the new channel which is decided as a best one by the decision module.

Several works has been proposed in the literature survey for vertical handoff decision making, which is categorized as five different approaches.

1) Traditional approach uses the Received Signal Strength (RSS) loss as a value to trigger the handoff event.

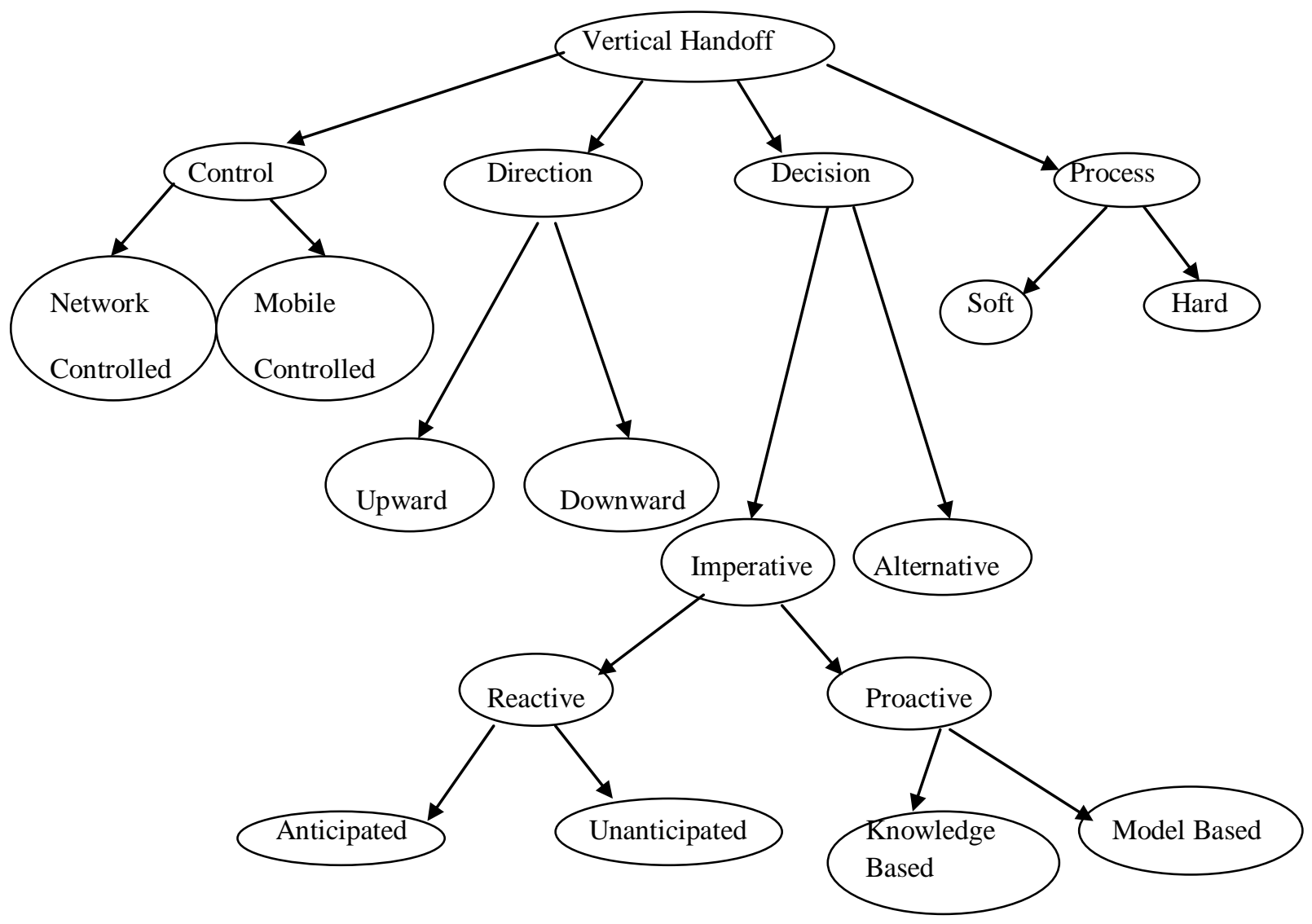

Figure 1. Classification of Vertical Handoff

But in the current communication environment the handoff can be triggered not only because of RSS loss but also the need for best network. Which means the handoff request will be initiated when the user offers best network for their application even the RSS signal strength is very strong. So the Traditional method is not suitable.

2) Next one is function-based which considers the cost function[4] as a measurement to choose the best network. The network with minimum cost is decided as a target network.

3) Multiple Attribute Decision Making (MADM) deals with the problem of choosing an alternative from a set of alternatives which are characterized in terms of their attributes. The most popular classical MADM methods are: SAW (Simple Additive Weighting)[5] The overall score of a candidate network is determined by the weighted sum of all the attribute values. TOPSIS[6] (Technique for Order Preference by Similarity to Ideal Solution): the chosen candidate network is the one which is the closest to ideal solution and the farthest from the worst case solution. AHP (Analytic Hierarchy Process)[7] decomposes the network selection problem into several sub-problems and assigns a weight value for each sub-problem. GRA[8] (Grey Relational 
Analysis) is then used to rank the candidate networks and selects the one with the highest ranking.

4) User-Centric[9] approach which mainly focuses on user preferences and non-real time applications.

5) A Fuzzy logic and Artificial Neural Networks[2] are used for decision making which uses intelligent techniques. In which fuzzy rule set is generated for decision making in fuzzy logic. In ANN the requirements are given as input, in the hidden layer decision is made based on the requirement and finally the selected network is given as output. Combination of these two techniques provides an excellent result.

6) The last one is based on the knowledge of context information of the mobile terminal and the networks to make efficient decision.

Apart from these, dynamic decision model, congestion based method, location based method, direction based handoff, load balancing methods, handoff decision using genetic algorithm and game theory has also been proposed in the survey. All these methods mainly use the parameters like RSS, bandwidth, delay, jitter, power, cost, velocity etc. Some papers use direction, location, network load and congestion. Only few papers consider handoff call dropping and call blocking. This paper proposes a frame work for decision making by considering call blocking and call dropping as a parameter.

\section{PARAMETERS FOR VHO DECISION MAKING AND PERFORMATION EVALUATION}

VHO parameters can be categorized into four different groups[10]. Network related parameters are Bandwidth, delay, RSS, SINR, Cost and Security. Device related parameters are Velocity, Power and Location. User related parameters are user preferences and application type requirements (real-time and non real-time applications). Service related parameters are cost and service capability.

Application types can be categorized into four types based upon the traffic. Each type requires certain QoS and some may be tolerable. Following table outlines the QoS requirements for all the traffic classes.

Table 2. Traffic classes and their QOS requirements

\begin{tabular}{|c|c|c|c|c|}
\hline Traffic & BER & Delay & Jitter & Bandwidth \\
\hline Conversational & $\begin{array}{c}\text { Need not } \\
\text { be Low }\end{array}$ & $\begin{array}{c}\text { Should be } \\
\text { Low }\end{array}$ & $\begin{array}{c}\text { Should be } \\
\text { Low }\end{array}$ & $\begin{array}{c}\text { Need not } \\
\text { be high }\end{array}$ \\
\hline Streaming & $\begin{array}{c}\text { Need not } \\
\text { be Low }\end{array}$ & $\begin{array}{c}\text { Should be } \\
\text { Low or } \\
\text { medium }\end{array}$ & $\begin{array}{c}\text { Should be } \\
\text { Low }\end{array}$ & $\begin{array}{c}\text { Should be } \\
\text { high }\end{array}$ \\
\hline Interactive & $\begin{array}{c}\text { Should be } \\
\text { Low }\end{array}$ & $\begin{array}{c}\text { Should be } \\
\text { Low or } \\
\text { medium }\end{array}$ & $\begin{array}{c}\text { Need not } \\
\text { be Low }\end{array}$ & $\begin{array}{c}\text { Should be } \\
\text { high }\end{array}$ \\
\hline Background & $\begin{array}{c}\text { Should be } \\
\text { Low }\end{array}$ & $\begin{array}{c}\text { Need not } \\
\text { be Low }\end{array}$ & $\begin{array}{c}\text { Need not } \\
\text { be Low }\end{array}$ & $\begin{array}{c}\text { Should be } \\
\text { medium at } \\
\text { least }\end{array}$ \\
\hline
\end{tabular}

To evaluate the performance of the decision making algorithm throughput, delay, jitter, data rate, error rate, packet loss, handoff call blocking rate, call dropping rate and number of handoffs triggered can be considered.

\section{CROSS LAYER BASED VHO DECISION MAKING FRAMEWORK}

Several works is going on using cross layer approach. Each work has its own strength and weakness. In cross layer approach[1], congestion control can allocate data rates without requiring precise prior knowledge of the capacity region because network jointly optimizes both the data rates of the users and the resource allocation at under lying layer. In this paper, handoff call dropping and call blocking, number handoffs where not addressed. Paper [13] explains that crosslayer solutions are mainly proposed for handoff management. It aims to achieve layer 3 handoff with help of layer 2. Layer 2 reports the signal strength and movement detection information in advance, the system can make better preparation for the network layer handoff so that the packet loss is eliminated and the handoff latency is reduced. But this paper did not consider the application type to choose the best network. Some other works for vertical handoff employing some form of cross-layer approach [3] [4] [13] [14] which uses a policy based information and others like presence and absence of beacon and data packets to offer advanced mobility support. User defined policies are used to decide the most suitable network to satisfy the user's expectations. In [13], location information is used to perform transition analysis in deciding handoffs. None of these approaches define a complete cross-layer platform for mobility management. If the mobility management is not properly done then in turn handoff management is also an unsuccessful one. Normally the heterogeneous network has physical layer, data link layer, network layer, transport layer and application layer. In which the Link Layer is responsible for handoff initiation by using Media Independent Handover protocol(MIH) , Network Layer provides the IP address of the mobile device which uses Mobile IP protocol which is used to consider the destination based vertical handoff , and Transport Layer is responsible for handoff execution while the Application Layer is responsible for selecting the network based on application. So that, when we do vertical handoff it is necessary to consider all these layer's information in order to select the best QoS network. Most of the existing work is not considering the cross layer information. Some papers take this method into account, but the author is not considering the Call Blocking and Call dropping as a parameter for handoff decision making. So this paper presents a framework which considers both cross layer based and call admission control based network selection method.

Information for decision making algorithm is obtained from different layers[11]. The Link Layer (LL) provides the information like RSS, packet error rate, link speed. Transport Layer (TL) is responsible to provide the details like connection characteristics, throughput, delay and jitter. Commercial network subscription details, user preferences and network selection are taken from Application Layer(AL). So that the cross layer based architecture suits for information gathering.

In the given architecture LL, TL and $\mathrm{AL}$ information are gathered and stored in the database which is used as input for Handoff Triggering (HT). The database is updated every time when there is a change in the given network characteristics. Because of the support of MIH protocol of LL, the RSS and link speed where came to know in advance, handoff delay and pocket loss can be reduced. 
Handoff initiation module initiates HT based on the information stored in the database. The Handoff Triggering event may occur when there is signal strength loss which is imperative handoff trigger or when there is a need for best network based on the requirement of user or application which is called as alternative handoff trigger. While making the handoff decision it should consider type of handoff, whether it is an imperative or alternative handoffs, based on that decision method will vary.
Handoff execution module, executes the handover function to the mobile node from old network to the selected network. The Handoff execution monitor monitors the currently selected network function in order to validate the decision made by the decision module. The result is forwarded to the decision module for future corrections.

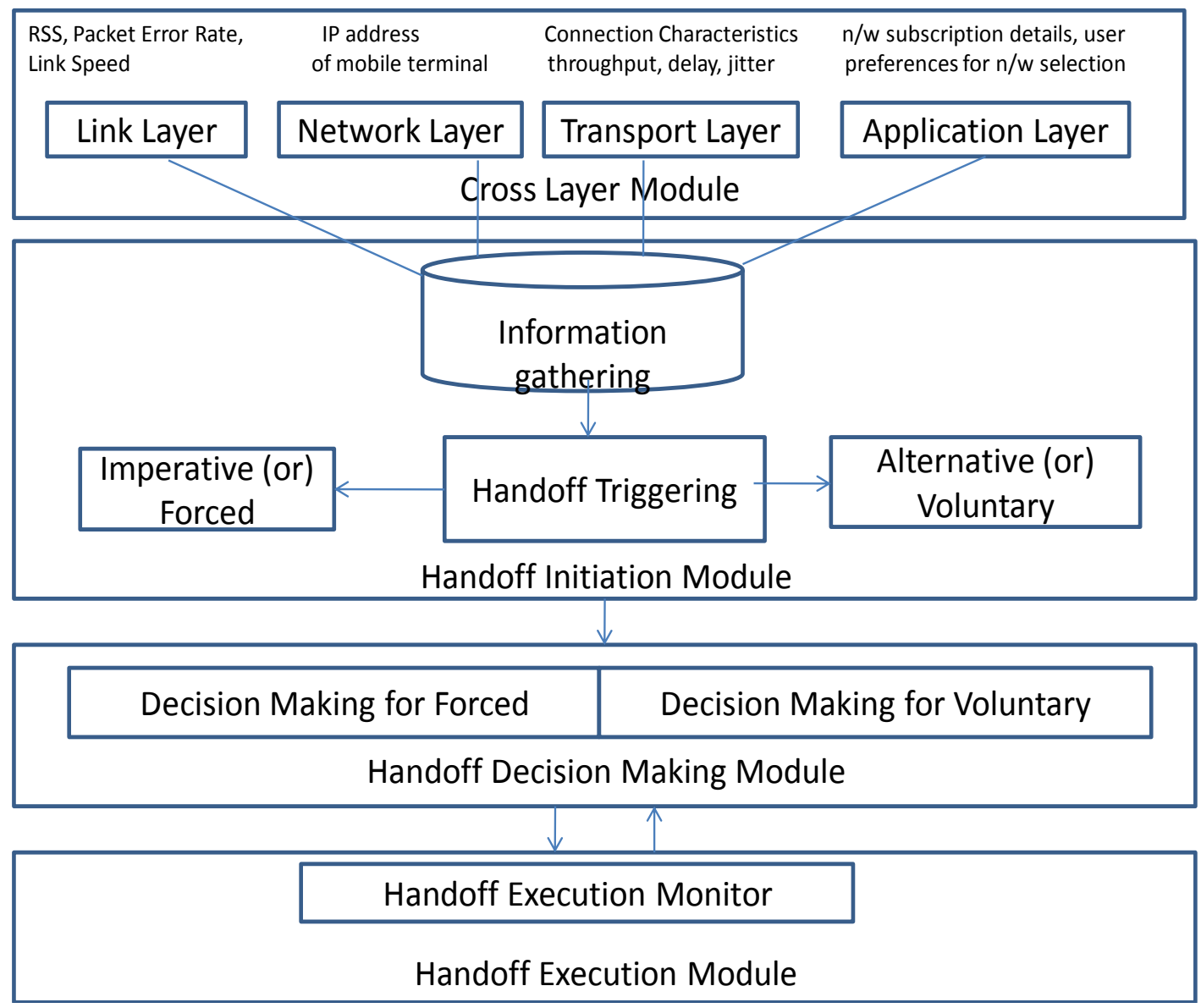

Figure 2. Cross Layer Based VHO Decision Making Framework

\section{CONCLUSION DIRECTION}

This paper presents an overview of handoff types, handoff needs and when it triggers and from where information was gathered. Based on the literature review it came to know the importance of cross layer based decision making. The frame work for VHO decision making based on cross layer approach has been proposed. Future plan is to propose an algorithm to make handoff decision based on this framework by considering new call arrival, handoff request, bandwidth and other parameters.

\section{REFERENCES}

[1] "Congestion-aware Proactive Vertical Handoff Decision Using Coalition Game", - S.V. Saboji, C. B. Akki, International Journal of Soft Computing and
Engineering (IJSCE) ISSN: 2231-2307, Volume-1, Issue6, January 2012

[2] “ Intelligent Proactive Handover and QoS Management using TBVH in Heterogeneous Networks" Fatema Sabeen Shaikh, January 2010, Middlesex University.

[3] "Vertical Handoffs in Fourth-generation Multinetwork Environments," - J. McNair and F. Zhu, IEEE Wireless Comm., vol. 11, no. 3, June 2004.

[4] "Policy-enabled handoffs across heterogeneous wireless networks", - H. Wang, R. Katz, J. Giese, Second IEEE Workshop on Mobile Computing Systems and Applications, 1999 (Proceedings WMCSA'99), 1999, pp. 51-60.

[5] "Vertical Handover decision schemes using SAW and WPM for Network selection in Heterogeneous Wireless Networks" - K.Savitha, Dr.C.Chandrasekar, Global 
Journal of Computer Science and Technology Volume 11 Issue 9 Version 1.0 May 2011

[6] "An Improved TOPSIS Vertical Handoff Algorithm for Heterogeneous Wireless Networks", - Liu Sheng-mei, Pan su, Xu Ming-hai, 2009.

[7] "AHP and Group Decision Making for Access Network Selection in Multi-Homed Mobile Terminals", - K. Radhika, Dr. A. Venugopal Reddy, International Journal on Computer Science and Engineering (IJCSE), ISSN : 0975-3397 Vol. 3 No. 10 October 2011

[8] "Grey Relation Analysis for Vertical Handover Decision Schemes in Heterogeneous Wireless Networks", - K. Savitha, C. Chandrasekar, European Journal of Scientific Research, ISSN 1450-216X Vol.54 No.4 (2011), pp.560568.

[9] "A Constrained MDP-based Vertical Handoff Decision Algorithm for 4G Heterogeneous Wireless Networks" Chi Sun . Enrique Stevens-Navarro - Vahid ShahMansouri - VincentW.S. Wong Received: 22nd December 2008 / Accepted: 10th January 2011.

[10] "Minimization of Number of Handoff Using Genetic Algorithm in Heterogeneous Wireless Network"Mrs.Chandralekha, Dr.Praffula Kumar Behera, nternational Journal of Latest Trends in Computing (EISSN: 2045-5364) 24 Volume 1, Issue 2, December 2010

[11] "A Cross-Layer User Centric Vertical Handover Decision Approach Based on MIH Local Triggers", Maaz Rehan, Muhammad Yousaf, Amir Qayyum, and Shahzad Malik, J. Wozniak et al. (Eds)., WMNC 2009, IFIP AICT 308, pp. 359-369, 2009. @ IFIP International Federation for Information Processing 2009.

[12] “A Survey Of Mobility Management In Next-Generation All-Ip-Based Wireless Systems",- Ian F. Akyildiz, Jiang
Xie, And Shantidev Mohanty, Georgia Institute Of Technology

[13] "Analysis of Handoff in a Location-Aware Vertical Multi-Access Network", - M. Ylianttila, J. Makela, and K. Pahlavan, Elsevier J Computer Networks, 2005, 4:185-201.

[14] "Vertical Handoffs in Wireless Overlay Networks", - M. Stemm and R. Katz, Mobile Networks and Applications, 1998, pp. 335-350.

\section{AUTHOR'SPROFILE}

A. Bhuvaneswari completed her Masters Degree in Computer Science and Master of Philosophy in Computer Science in the years 2002 and 2005 respectively. She has 10 years of teaching experience at Cauvery College for Women , Trichy, Tamil Nadu, India. Currently she is doing her research in the area of Mobile Communication under the guidance of Dr.E.George Dharma Prakash Raj .

Dr.E. George Dharma Prakash Raj completed his Masters Degree in Computer Science and Masters of Philosophy in Computer Science in the years 1990 and 1998. He has also completed his Doctorate in Computer Science in the year 2008. He has around twenty-one years of Academic experience and thirteen years of Research experience in the field of Computer Science. Currently he is working as an Asst.Professor in the Department of Computer Science and Engineering at Bharathidasan University, Trichy, India. He has published several papers in International Journals and Conferences related to Computer Science and has been an Editorial Board Member, Reviewer and International Programme Committee Member in many International Journals and Conferences. He has convened many National and International Conferences related to Computer Science. 